\title{
The magnetic metrology of materials-A review
}

\author{
J SIEVERT and H AHLERS \\ Physikalisch-Technische Bundesanstalt, Braunschweig, Germany
}

\begin{abstract}
This paper gives a survey of the present state and new aspects of the measurement techniques for the characteristics of magnetic materials (except for recording materials) with several examples. Particular attention is paid to the aspects of reproducibility and traceability to SI units, and to the current trend of quality assurance and standardization.
\end{abstract}

Keywords. Magnetic materials; measurement techniques.

\section{Introduction}

A great variety of methods for the determination of the magnetic properties of materials is in use. The characteristics of the methods vary with the purpose (research, calibration, acceptance tests or quality control measurements), the sample's properties (shape, size, permeability, etc.), and the quantities to be measured (coercivity, remanence, saturation magnetization, permeability, anisotropy, magnetic losses, maximum energy product, magnetoelastic properties, etc.).

Table 1 lists the main materials (except recording materials which we shall not treat in this paper) in use together with the quantities of interest. Most of the measurement methods for their determination are well known, and experience has been gained in using them. However, modern electronics has given an impetus to the development of rationalized as well as more complicated measurement techniques. Today's powerful computers and finite element method (FEM) programs make a more detailed estimate of the error characteristics of the magnetic circuits used for the measurements possible, thus bringing about modifications of traditional methods.

The following survey reports, with the help of examples because completeness is impossible within the frame of this paper, on fundamental aspects for the measurements in DC and AC fields, tracing to the SI units, and standardization. We will focus on the methods for characterization of the properties relevant for applications rather than basic physical characteristics such as domain structure, Mössbauer spectrum, etc.

\section{Basic aspects, tracing to SI units}

In principle there are three metrological concepts one of which is given priority depending again on the purpose (see table 2). In magnetic materials metrology, the repeatability concept reigns in materials development and research labs, reproducibility where trade is involved, and, most rarely, accuracy where absolute values or natural constants are aimed at.

The main quantities for the determination of magnetic material properties are two: the magnetized state, i.e. the magnetic polarization $J$, and the driving force necessary 
Table 1. Main categories of magnetic materials and the most important quantities to be measured.

\begin{tabular}{|c|c|c|}
\hline Soft & $\begin{array}{c}\text { Hard } \\
\text { magnetic materials }\end{array}$ & Feebly \\
\hline $\begin{array}{l}\text { Electrical steel } \\
\text { NiFe } \\
\text { Soft ferrites } \\
\text { Amorphous }\end{array}$ & $\begin{array}{l}\text { AlNiCo } \\
\text { Ferrites } \\
\text { RECo } \\
\text { NdFeB }\end{array}$ & $\begin{array}{l}\text { Para- and diamagn. } \\
\text { materials Stainless steel } \\
\text { Brass, Copper }\end{array}$ \\
\hline $\begin{array}{l}\text { Saturation polarization } J_{s} \\
\text { DC hysteresis loss } \\
\text { AC power loss and } \\
\text { apparent power } \\
\text { Rotational power loss } \\
B(H) \text { curve } \\
\text { Magnetostriction }\end{array}$ & $\begin{array}{l}\text { Remanence } \\
\text { Coercivity } \\
(B H)_{\max } \\
\text { Stability }\end{array}$ & Susceptibility \\
\hline
\end{tabular}

Table 2. Metrological concepts and their priorities.

\begin{tabular}{ll}
\hline Concept & Priority with \\
\hline Repeatability & $\begin{array}{l}\text { Materials research and optimization } \\
\text { Reproducibility }\end{array}$ \\
$\begin{array}{l}\text { Methods laid down in standards, quality } \\
\text { control, acceptance tests, intercomparisons }\end{array}$ \\
Accuracy & $\begin{array}{l}\text { Realization of units, absolute characteristics } \\
\text { of materials, natural constants }\end{array}$ \\
\hline
\end{tabular}

to reach that state, i.e. the magnetic field strength. Table 3 lists the main methods for the measurement of these two quantities. However, only a few of the $H$ methods are applied to materials, viz. Hall probes, and, most of all, inductive methods. Besides these, the indirect method of determining $H$ from the magnetizing current is very common in the cases where the demagnetizing field is small (closed magnetic circuit) or is well known (ellipsoidal specimen). The inductive method requires integration of the signal. The magnetic field strength $H$ inside the specimen can be measured approximately only close to its surface. These points will be considered in the next section.

Recently, tracing the magnetic units to the SI system of units is considered more and more important. The ISO 9000 standards system is increasingly applied for quality assurance not only in industrial quality control but also in research labs. The laboratories authorized for the realization, maintenance and dissemination of the national units and their tracing to the international level are national laboratories such as the NPL in New Delhi or the PTB in Braunschweig, Germany. In our field of magnetic materials metrology, the tracing to SI units is in no way a problem of the electrical and mechanical units forming the basis for our derived magnetic units because those are known to a much higher accuracy than that to which material properties are, in view of their scatter, definable. The magnetic circuit is mostly the main problem. 
Table 3. Survey of the most important methods for the measurement of magnetic field strength $H$ and magnetic polarization $J$. (for details and references see Ahlers and Sievert (1991), Weyand (1991) and Zijlstra (1967)) and for the study of the magnetic structure of the material. (Less known abbreviations: SST, single sheet tester; VSM(VCM), vibrating sample (coil) magnetometer).

$\begin{array}{ll}\text { Magnetic field strength } H: & \begin{array}{l}\text { Magnetic polarization J: } \\ \text { (Material characteristics) }\end{array} \\ \begin{array}{l}\text { Induced voltage } \\ \text { Rotating coil, search coil }\end{array} & \begin{array}{l}\text { Force } \\ \text { Gouy and Faraday balance; } \\ \text { torque, torsion, vibrating reed } \\ \text { magnetometer }\end{array} \\ & \text { Induced voltage } \\ \text { Indirect measurement } & \text { Ring core, Epstein, SST, } \\ \text { From magnetizing current } & \text { permeameter, VSM, } \\ \text { (defined magn. circuit) } & \text { SQUID, VCM } \\ & \text { Others } \\ \text { Galvanomagnetic sensors } & \text { Astatic magnetometers } \\ \text { Hall effect. magnetoresistance, } & \text { Hall probe, fluxgate or torsion } \\ \text { magnetotransistors, etc. } & \text { sensor, Faraday rotation, Kerr } \\ & \text { effect, ESR, Mössbauer }\end{array}$

Ferromagnetic sensors

Fluxgate, thin-film, magnetostrictiveoptoelectronic sensors (optical fibres)

Quantum effect sensors

NMR, ESR, Zeeman effect,

Faraday rotation,

RF- and DC-SQUID

\author{
Magnetic polarization $J$ : \\ (Material characteristics) \\ Gouy and Faraday balance; \\ torque, torsion, vibrating reed \\ magnetometer \\ Induced voltage \\ permeameter, VSM, \\ UID, VCM \\ Astatic magnetometers \\ sensor, Faraday rotation, Kerr \\ effect, ESR, Mössbauer
}

For the calibration of field strength measuring devices, almost only the nuclear magnetic resonance (NMR) technique is applied, making use of the results of precision fundamental experiments for the determination of the gyromagnetic coefficient of the proton (Weyand 1991). This follows the general trend of basing all units on fundamental constants and quantum effects. Commercial NMR instruments are available, allowing, when due caution is exercised for field homogeneity and equivalence, an accurate calibration of secondary standards such as $H$ sensing coils or Hall probes.

The calibration of polarization measuring devices depends on their principle. Force sensing devices can be calibrated with the help of a reference mass. The most-in-use inductive method requires, besides the calibration of the search coil, calibration of the flux integrator. Formerly the ballistic galvanometer was in common use as the flux integrator whereas nowadays electronic integrators are preferred. Fluxmeters are calibrated either by means of a mutual inductor which is calibrated itself electrically, or otherwise by means of a capacitor discharge. Modern electronics have opened a more convenient and precise solution to this problem. The electronic calibrator developed by Rahf (1989) which in turn is calibrated by precision tracing to time and voltage standards, generates trapezoidal voltage $\times$ time curves with controllable slope and duration, and allows the calibration of fluxmeters to better than $2 \times 10^{-4}$. 
In order to avoid the effort of tracing back the indication of a magnetometer directly to SI units, reference samples, in particular nickel samples, are applied (Sievert et al 1990a). The saturation polarization of $\mathrm{Ni}$ is known accurately from earlier extensive investigations (Bennet et al 1978; Graham 1982) and amounts to $0.615 \mathrm{~T} \pm 0.0005 \mathrm{~T}$ at $23^{\circ} \mathrm{C}$. We studied the error sources for the case of cylindrical Ni reference samples used in the air gap of an iron core magnet. The calibration of such cylindrical reference samples which are available from the Bureau of Reference of the EU (Commission of the European Union, Dir. Gen. Science, Research and Development, Bureau BCR, Rue de la Loi 200, B 1049 Brussels, Belgium) can be carried out with a relative uncertainty of $0.15 \%\left(\sigma_{1}\right)$, and the subsequent calibration procedure in laboratories using these samples should be possible with a relative uncertainty of $0.5 \%$ (Sievert et al 1990a).

\section{Excitation, sensors and measurement of the electric signal}

Measuring magnetic materials involves four partial problems (see also figure 1 with the exemplary ring specimen):

- excitation of the specimen with specific conditions determined by the specimen's properties and geometry and for DC or AC fields;

- type of sensors, their geometry and position;

- the electrical measurement including integration;

- the evaluation.

The first two points refer to the magnetic circuit. We distinguish between open (samples in solenoids, superconducting or Bitter magnets, spherical sample in an electromagnet) and closed (ring cores, Epstein frame, sheet samples in yokes, cylinders clamped between the poles of an electromagnet, etc. (for ref. see e.g. table 4, 404-4,

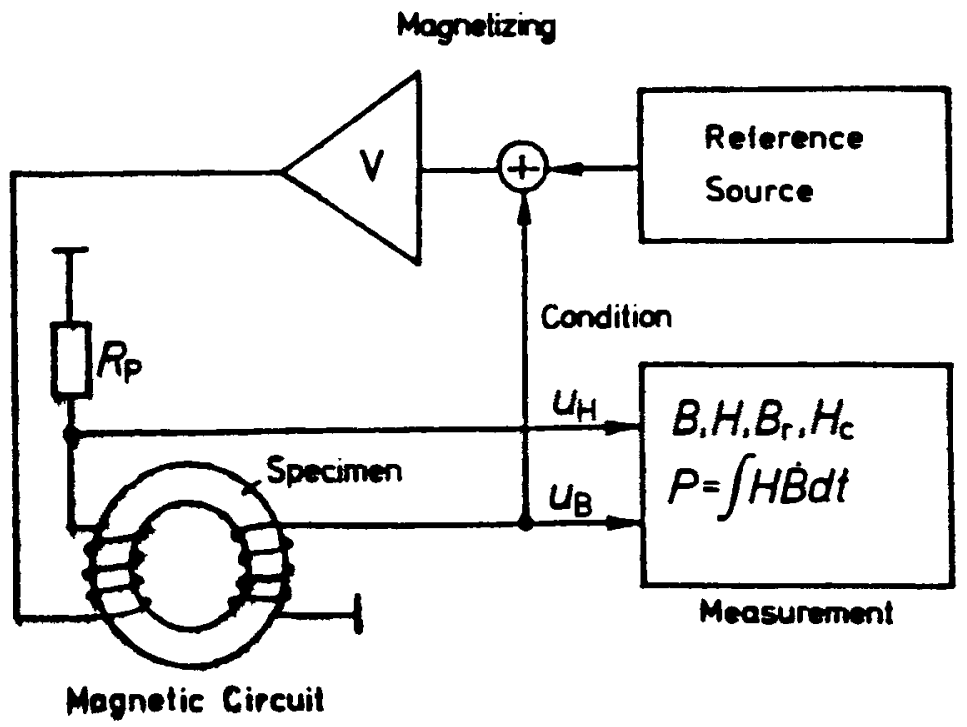

Figure 1. Basic elements of a magnetic circuit for measuring magnetic materials. 
Table 4. IEC standards on methods of measurement for the magnetic properties of materials (abbreviated titles).

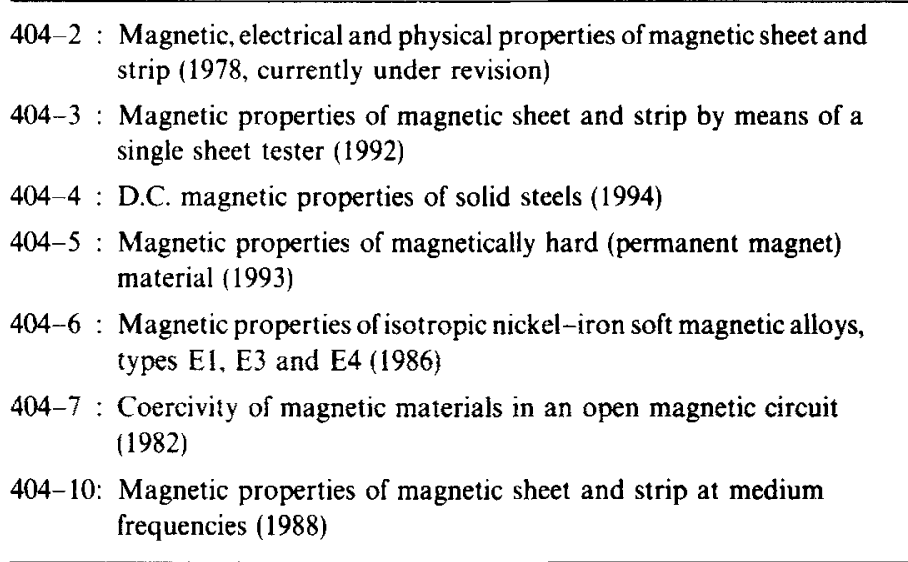

$-2,-3$, and -5 , respectively)) magnetic circuits. The methods of exciting and sensing depend decisively on whether we have an open or closed magnetic circuit.

DC measurements on small ferromagnetic and on feebly magnetic samples are preferably carried out in an open magnetic circuit applying force (Gouy or Faraday balance) or inductive methods for sensing of the polarization. In iron-free circuits, the field strength is conveniently determined from the magnetizing current and the coil constant of the magnetizing coil, whereas in iron core magnets usually a separate sensor for the field has to be used.

The closed magnetic circuit is the one preferably used where quality control is aimed at and averaging over a greater amount of material is requested. Thus most of the magnetic standards treat the closed circuit (see table 4). The following considerations essentially hold for the DC and AC case equally.

The closed magnetic circuit may consist completely of the specimen (ring cores or Epstein specimens). In this case $H$ can easily be determined from a measurement of the magnetizing current on the basis of the simplified Ampere's law: $H=N_{1} \cdot I_{1} / l_{\text {eff }}$, with $N_{1}$ number of turns of the magnetizing winding and $I_{1}$ magnetizing current. The effective magnetic path length $l_{\text {eff }}$ is, in particular in the less homogeneous Epstein frame, an approximation for the path along which the magnetic potential $N_{1} \cdot I_{1}$ drops. In so far this simplification always implies a systematic error. Epstein results are, for instance, too high by about $4 \%$ for low inductions and, for a high induction, too low by $4 \%$ to $10 \%$, depending on the permeability of the sample (Sievert 1984).

In other setups the specimen may make up only a part of the magnetic circuit (single strip (or sheet) testers, permeameters, see table 4, 404-3 and -4 , for reference), fixed between flux closure yokes.

In this case also the field strength can be determined from the magnetizing current, if the magnetic potential dropping along the path through the yokes can be neglected (Sievert et al 1990b). However, where relatively high fields are to be reached as in permeameters and electromagnets, tangential $H$ sensing coils or also Hall probes are applied for the measurement of $H$ at the sample's surface. This method involves, in principle, a systematic error which can be considerable, if the magnetizing winding is wound on the yokes. However, if the winding surrounds the specimen uniformly, 
air gaps are kept small, and highly permeable yokes provide a small field gradient transverse to the specimen's surface, this error may be small. It increases with the permeability of the sample, and thus depends on the flux density inside the sample. In special cases such as two-dimensional excitation and measurement on steel sheet samples where the conditions mentioned before cannot be met, double $H$ coils for extrapolation to the surface may be necessary, or Rogowski-Chattock potential sensing coils (Zijlstra 1967) can be used to come closer to the surface. Calculating the field distribution by means of FEM for the area where the sensors are to be located is often a very valuable help for the assessment of the accuracy of the sensing.

As to the homogeneous and defined magnetizing of the sample, certain conditions must be met. In the DC case which, in fact, is usually a quasi-DC exciting, the change of the field strength must be controlled to be sufficiently slow in order to avoid eddy currents. This can be obtained with the help of a feedback which provides constant steps of the flux density change (Ahlers and Hartwig 1985). For permanent magnet material with very high coercivity such as $\mathrm{NdFeB}$, separate pulse magnetizers or superconducting magnets must be used for the initial magnetizing up to saturation. Thereafter the sample is to be moved into the sensing coil system whereas the measuring device (flux integration) must already be working simultaneously. In the AC case, the magnetic loss in the sample depends on the distribution of higher harmonics on the $H$ and $J$ signals which in turn depend on the ratio of the output impedance of the power supply to the input impedance of the magnetizing circuit. It is therefore conventionally agreed that the flux density is to be changed sinusoidally (which means using a voltage source), which can be made, for instance, by an electronic negative feedback. It corresponds to a form factor value of the $J$ signal of $1 \cdot 1107$. This condition can hardly be met for high flux densities where the power supply reaches its current limit. Deviations from symmetry of the flux density involve even harmonics and lead also to considerable errors (Ahlers and Sievert 1984).

Modern electronics are a decisive help in meeting the conditions required for magnetizing the sample. However, still more they are essential for due signal treatment and evaluation. After, in a first step, analog electronics had entered magnetic metrology, there is nowadays no alternative to digital procedures which were first introduced by Capptuller et al, if one wishes to measure efficiently, fast and precisely (Capptuller 1971; Capptuller and Ahlers 1975). By this we understand, in the DC case, voltageto-frequency conversion and then counting of the signal which includes the integration process, and in the AC case, analog-to-digital conversion of the signal from the sensors and digital evaluation in a computer. The main advantages are the following: easy to calibrate using a precise DC voltage source, absolutely linear and time-independent integration and multiplication of the power measurement. However, for power measurements one still has to check that the preamplifiers do not change the phase angle which may, where the power factor is low, cause considerable errors.

\section{Standardization}

Methods to be standardized should be as simple and reproducible as possible. Sometimes research activities and intercomparisons precede the publication of a standard (Sievert 1984; Sievert et al 1990b). Table 4 presents a survey of existing IEC standards in the field of magnetic materials métrology. Naturally in this list methods 
used for quality tests of economically important materials are predominant. Thus magnetic steel sheet plays the main role. Fourteen of the 40 national members of IEC take part in preparation and repeated revision of these standards. Other important standard systems comprising magnetic standards are the ASTM (US), BS (UK), DIN (Germany), EN (European Union), GOST (former USSR) and JIS (Japan).

\section{References}

Ahlers H and Sievert J 1991 in Landolt-Börnstein (eds) J Bortfeldt and B Kramer (Berlin) New Series, vol. la: "Units", section 2.3.3.2

Ahlers H and Hartwig H 1985 Proc. 7th EPS Conf. on Soft Magnetic Materials (SMM7) (ed.) Blackpool (Cardiff) p. 26

Ahlers H and Sievert J 1984 PTB-Mitt. 9499

Bennett L H, Page C H and Swartzendruber L J 1978 J. Res. Nat. Bur. Sta. 839

Capptuller H 1971 IEEE Trans. Instrum. Meas. IM-20 265

Capptuller H and Ahlers H 1975 Proc. Int. Colloq. Electronique Mesure (Paris) p. 607

Graham C D 1982 J. Appl. Phys. 532032

Rahf L. 1989 J. Magn. Magn. Mater. 83541

Sievert J, Ahlers H, Siebert S and Enokizono M 1990a IEEE Trans. Magn. MAG-26 2052

Sievert J 1984 IEEE Trans. Magn. MAG-20 1702

Sievert J, Binder M and Rahf L 1990b (SMM9 Conference, Madrid 1989) Anales Fisica B86 76

Weyand K 1991 in Landolt-Börnstein (eds) J Bortfeldt and B Kramer (Berlin) New Series, vol. la: "Units" section 2.3.3.1

Zijlstra H 1967 in Series of monographs on selected topics in solid state physics (ed.) E Wohlfahrt (Amsterdam: North-Holland) 